\title{
Developing multi-sensor drones for geological mapping and mineral exploration: setup and first results from the MULSEDRO project
}

\author{
Björn Heincke ${ }^{* 1}$, Robert Jackisch², Ari Saartenoja ${ }^{3}$, Heikki Salmirinne ${ }^{4}$, Sönke Rapp ${ }^{5}$, \\ Robert Zimmermann ${ }^{2}$, Markku Pirttijärvi ${ }^{3}$, Erik Vest Sörensen ${ }^{1}$, Richard Gloaguen ${ }^{2}$, Lisa Ek ${ }^{6}$, \\ Johan Bergström ${ }^{6}$, Arto Karinen ${ }^{3}$, Sara Salehi', Yuleika Madriz ${ }^{2}$ and Maarit Middleton ${ }^{4}$
}

REVIEW ARTICLE | OPEN ACCESS

GEUS Bulletin Vol 43 | e2019430302 | Published online: 29 July 2019

https://doi.org/10.34194/GEUSB-201943-03-02

The use of Unmanned Aerial Systems (UAS), also known as drones, is becoming increasingly important for geological applications. Thanks to lower operational costs and ease of use, UAS offer an alternative approach to aircraft-based and ground-based geoscientific measurements (Colomina

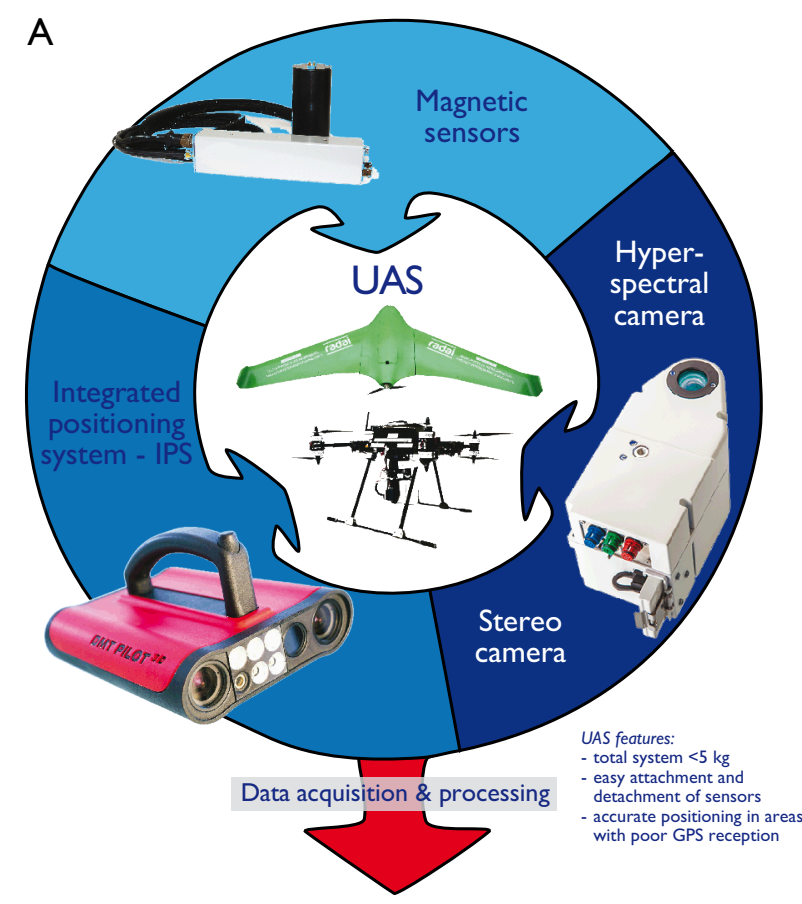

B Integrated physical property maps produced by combining DSM, hyperspectal and magnetic images
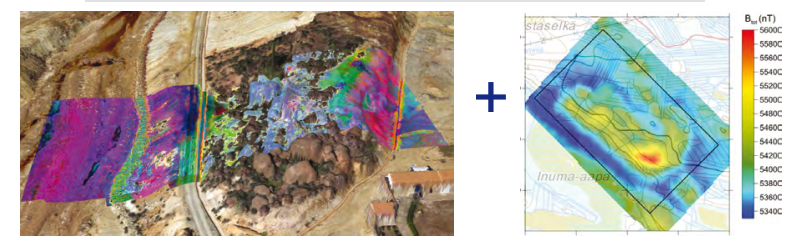

\& Molina 2014). Magnetic and hyperspectral UAS surveys hold particular promise for mineral exploration, and several groups have recently published studies of magnetic data collected by UAS for such applications (Malehmir et al. 2017; Cunningham et al. 2018), although equivalent studies using hyperspectral data are still rare (Kirsch et al. 2018). Combining both techniques is particularly useful. Magnetic measurements play an important role in mineral exploration, since magnetisation in rocks is mainly associated with magnetite and other iron minerals, which can be used in mapping and targeting of mineral deposits (Dentith \& Mudge 2014). Hyperspectral imaging (HSI) is a powerful exploration and mapping technique in areas where the rock surface is well-exposed, and where geological units and mineral compositions can be estimated from spectral features of the electromagnetic spectrum in the visual and infrared range.

This paper reports on the setup of UAS multi-sensor systems that can collect both magnetic and hyperspectral data, developed within the EU-funded MULSEDRO project (MULti-SEnsor DROnes). We focus on small, lightweight solutions with take-off weights less than $5 \mathrm{~kg}$. This is because regulations restrict commercial UAS operations in many countries according to take-off weight, flight height and operating range - typically, only operations in the (extended) visual line of sight are allowed. Lightweight systems are also particularly advantageous to support geological field campaigns as they can be deployed quickly to survey areas of interest.

Here, we use both a multi-copter and a fixed-wing UAS as both platforms have advantages. Multi-copters can fly at

Fig 1. In the multi-sensor drone (MULSEDRO) project we are developing A: UAS multi-sensor systems to gather both magnetic and hyperspectral data. B: These data are integrated with digital surface models (DSMs) to map physical properties on the ground. 
low elevation and speed, allowing them to follow strongly varying topography and collect high-resolution data that are comparable to traditional ground surveys. Fixed-wing UAS reach higher speeds and have longer endurance, such that larger areas can be mapped in a short time - comparable to smaller helicopter surveys. In addition, we have developed a new integrated positioning system (IPS) for UAS use that is not based on GPS. This affords accurate positioning within areas of poor GPS reception, such as mining tunnels and narrow valleys. The general concept of MULSEDRO is summarised in Fig. 1.

\section{Multi-sensor UAS systems}

The fixed-wing system is developed by the company Radai $O y$, Finland. The main characteristic of their self-constructed planes (Fig. 2A) are long flight times (up to three hours), and smooth flight trajectories, which are important for the quality of the acquired magnetic and multispectral data. The multi-copter system is developed by the Helmboltz Institute Freiberg for Resource Technology (HZDR-HIF), using a customised UAS and frames, on which different sensors can be quickly attached and detached (Fig. 2B) for efficient and flexible surveying. In both platforms, integrated Global Navigation Satellite Systems (GNSS) receivers and Inertial Measurement Units measure the positions and orientations of the UAS.

\section{Magnetic}

Both the fixed-wing and the multi-copter system were equipped with three-component fluxgate magnetometers, and magnetic base stations were set up to correct for the diurnal variations of the Earth's magnetic field. Flight operations are performed autonomously along a predefined survey path of in- and cross-lines. Barometrical sensors together with a digital elevation model are used to keep the flight altitude relative to the ground as constant as possible. Minimum operational altitudes for fixed-wing and multi-copter operations are typically governed by safety margins and terrain undulations, and can be as low as $c .30 \mathrm{~m}$ and $c .15 \mathrm{~m}$, respectively. Typical line spacing for the fixed-wing surveys of 20-50 m are comparable with those of small scale helicopter surveys. However, with the multi-copters, we have already performed surveys with line spacing of only $7 \mathrm{~m}$, resulting in magnetic maps with resolutions comparable to groundbased magnetic studies. Software developed by Radai $O y$ is especially adapted to process magnetic data from UAS and includes advanced steps, e.g. Equivalent Layer Modelling (Pirttijärvi 2003) to reduce artefacts and noise, and to compute consistent magnetic fields for a constant altitude.

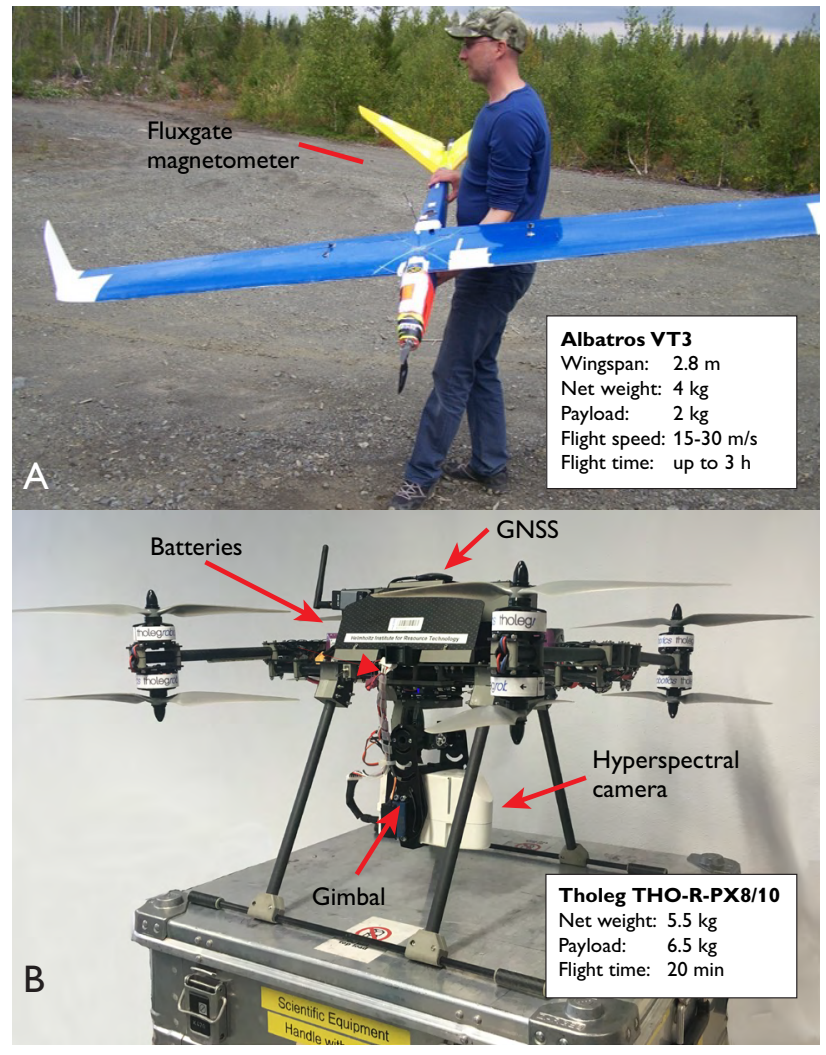

Fig 2. The UAS systems used within the project. A: Custom built fixed-wing UAS from Radai Oy equipped with a magnetometer. B: Customised multicopter from HZDR-HIF equipped with a hyperspectral frame camera.

One potentially problematic aspect of deploying magnetic sensors on multi-copters is that the electric motors can cause significant electromagnetic noise due to the proximity to the magnetic sensor (at distances $<1 \mathrm{~m}$ ). However, we observe that most noise in our magnetic surveys (using the THO-R$P X 8 / 10$ multi-copter from THOLEG) occurs at high frequencies and is filtered out efficiently during processing.

\section{Hyperspectral imaging and Photogram- metry}

Hyperspectral data are collected with the multi-copter platform using a frame-based snapshot camera (the Rikola Hyperspectral Imager camera from Senop $O y$ ). This instrument captures images in the visible and near-infrared part of the electromagnetic spectrum $(504-900 \mathrm{~nm})$ with image integration times of 1-2 s. Because of the significant integration time, flying at relatively high speed would cause spatial shifts of the spectral bands within a scene (e.g. assuming a typical band integration time of $15 \mathrm{~ms}, 50$ bands, and a typical multi-copter speed of $5 \mathrm{~m} / \mathrm{s}$, shifts between the first and last band of a scene (i.e. the area covered by the image stack) would be $>3.5 \mathrm{~s}$ ). This means that illumination conditions 

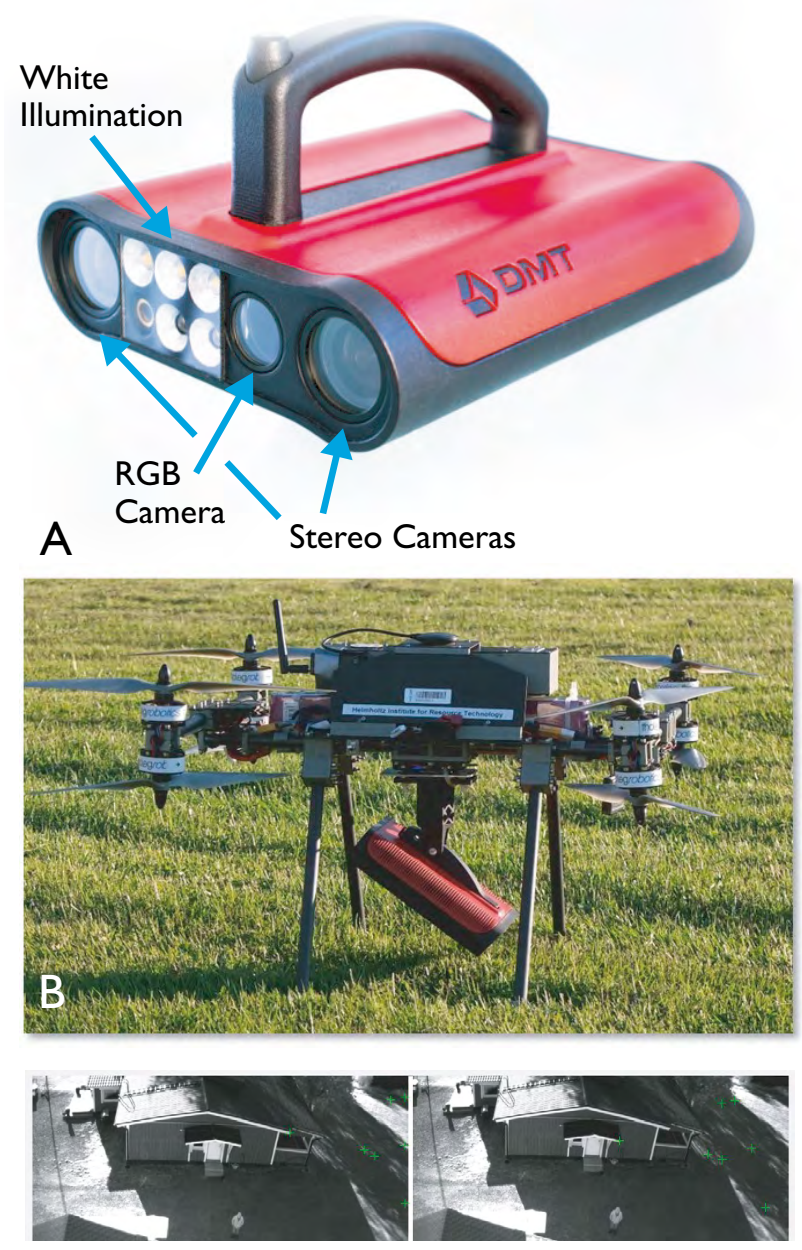

Fig 3. The integrated positioning system PILOT3D as A: handheld device. B: Mounted on a UAS. C: Images obtained by the stereo camera during a UAS test flight.

would significantly change for bands of one scene which again could result in inconsistent hyperspectral products for interpretation. Therefore, the multi-copter maintains a steady position in the air during data acquisition such that spectral bands of the same image strongly overlap and have little illumination changes.

During flight operation, hyperspectral data are usually collected using a setting of 50 image bands with a spectral resolution of $c .8 \mathrm{~nm}$ and operation heights in the range of 50-100 m, which results in ground pixels resolutions of $c$. 3-7 cm. To drape hyperspectral images on a precise surface topography, we performed an additional UAS survey to determine a high-resolution digital surface model (DSM) that covers the entire study area. A commercial fixed-wing equipped with a RGB camera is used for this survey and the DSM is determined by structure-from-motion photogrammetry using a flight pattern of parallel lines. We used the $M e$ physto toolbox (Jakob et al. 2017) to pre-process the HSI data and for integration with the DSM, which includes lens cor- rection, co-registration (correcting for spatial mismatches of spectral bands), orthorectification (matching the hyperspectral images with orthophotos - aerial photographs, geometrically corrected such that its scale is undistorted - obtained by photogrammetry), topographic correction (reducing the influence of topography onto the local illumination), and merging of images to a HSI mosaic and conversion from radiance to reflectance.

One limit of the current setup is that the spectral range of available lightweight hyperspectral sensors $(<2 \mathrm{~kg})$ mountable on UAS is restricted to the visible near infrared. However, diagnostic absorption features of most minerals are located at higher wavelengths, in the short-, mid- and long-wave infrared parts of the spectrum. Therefore, the MULSEDRO setup is currently limited to mapping iron-bearing minerals such as hematite, jarosite and goethite that have characteristic spectral features in the visible near infrared. The system can also identify vegetation features that can be used as proxies for mineralisation (i.e. different species of vegetation that correlate with the underlying rock types; Gupta et al. 2018). Other potential applications include mapping of rare-earth elements and monitoring mine tailings (Jackisch et al. 2018).

\section{Integrated Positioning system}

The IPS for UAS is developed from the handheld PILOT ${ }^{3 D}$ device (Fig. 3A) for underground mine navigation and 3Ddocumentation. The IPS is based on a multi-sensor approach, where an inertial measurements unit (a device typically used to manoeuvre aircraft) is combined with a stereo camera (Benecke et al. 2016). Both sensors independently acquire positioning information and combining these data from the devices provides accurate real-time positions with accuracies of $c .0 .1 \%$ of the distance covered at walking speed (c. 1-2 $\mathrm{m} / \mathrm{s}$ ). We have completed initial tests from drones (Figs 3B, C) to evaluate the performance of the IPS on faster moving platforms.

\section{Application and outlook}

The MULSEDRO UAS systems were tested during field campaigns in two mining areas of Central Finland (Fig. 4A) in the summers 2017 and 2018. Here, we present some of the initial results from one of these sites located in the northern part of the Siilinjärvi carbonatite-glimmerite complex $\left(63^{\circ} 08^{\prime} 44^{\prime \prime} \mathrm{N}, 27^{\circ} 44^{\prime} 16^{\prime \prime} \mathrm{E}\right), 1 \mathrm{~km}$ north of a large open pit phosphate mine. The complex intruded into granite gneiss $(2610 \pm 4 \mathrm{Ma})$ and covers an area $c .16 \times 1.5 \mathrm{~km}$ (O’Brien et al. 2015). Our objectives were to (1) trace the main subvertical carbonatite veins in areas covered by overburden by magnetic surveys, (2) identify relationships between those 

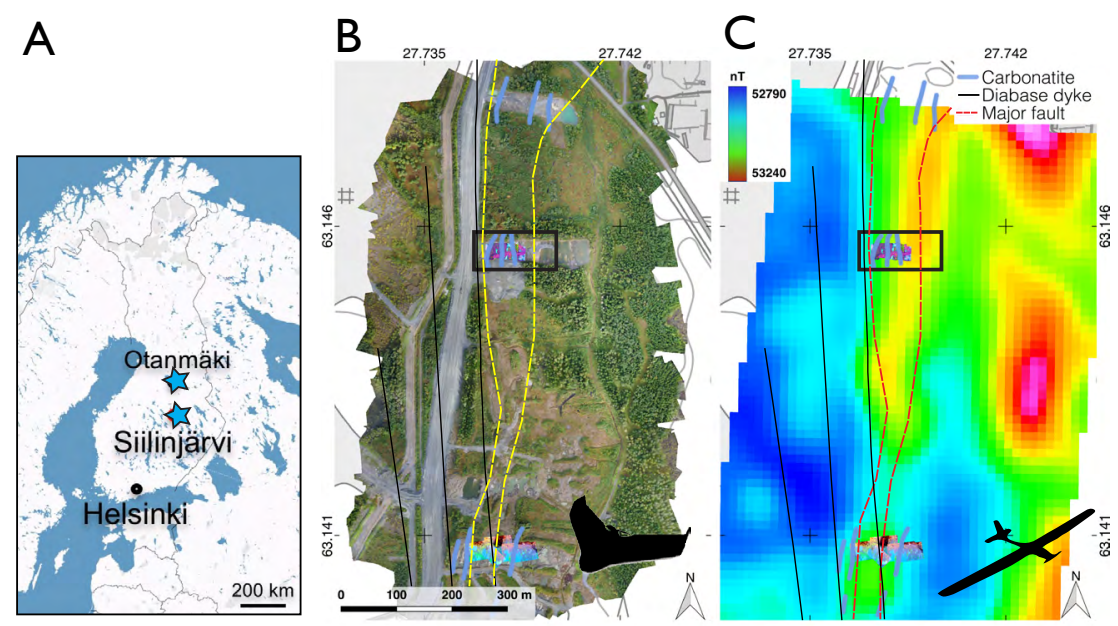

Fig 4. A: Location of field test sites in Finland. Note that only results from Siilinjärvi site, but not from Otanmäki site are presented here. B: Orthophoto from photogrammetry. C: Total magnetic intensity maps derived from fixedwing UASs surveys of the Siilinjärvi test site. D: Image from a processed hyperspectral multicopter-based data set draped on the digital surface model from photogrammetry. The hyperspectral image shows the combination of bands $3,2,1$ of a minimum noise fraction transformation in RGB, where green shading is associated with mainly fenite-hosting rocks. Location of the hyperspectral survey is outlined in B and $C$ with a black rectangle. (A second survey was performed in the southern end of the field site (see B and C), but these data are not presented

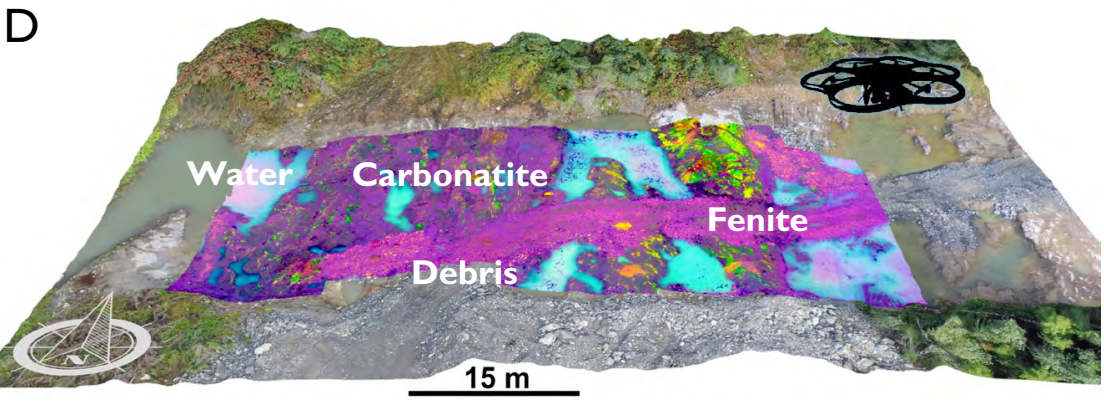
here). E: Ground sampling for one of the outcrops covered with hyperspectral surveying.

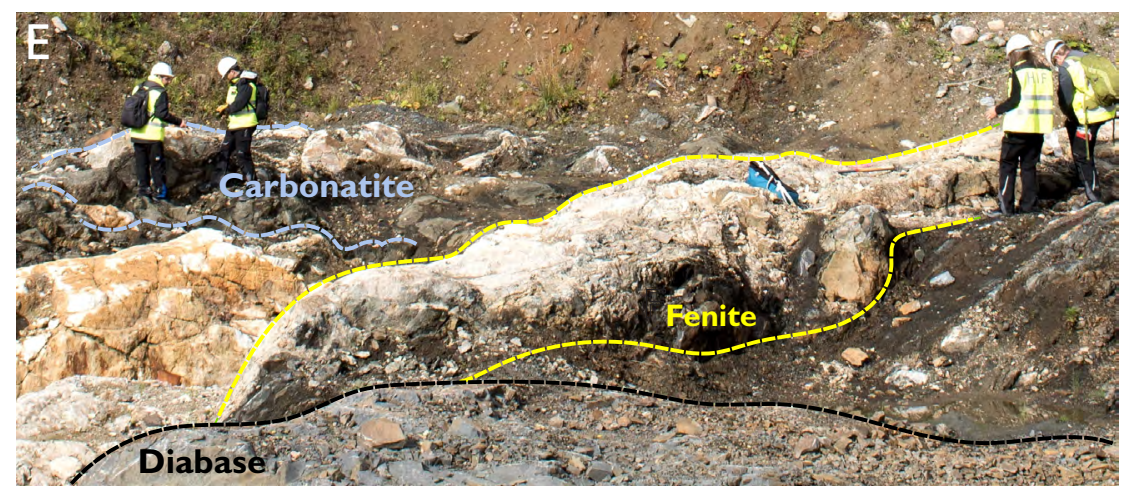

veins and the main mineralogy of the area, and to (3) identify possible indications of rare-earth elements in excavated test outcrops by HSI.

We conducted fixed-wing magnetic and photogrammetry surveys (Figs 4B, C) across areas of $c .1 \mathrm{~km}^{2}$. These surveys provide a larger-scaled picture of the topography and the magnetic anomalies from areas that are predominately covered by soil and vegetation. On accessible outcrops located within these areas, multi-copters equipped with hyperspectral and magnetic sensors collected data with significantly higher resolution (Fig. 4D) - down to a few centimetres for hyperspectral data. To connect these magnetic anomalies and spectral characteristics with rock properties on the ground, the UAS measurements were supplemented with ground-based investigations, including handheld XRF, spectroradiometry, magnetic susceptibility measurements and collection of rock samples for analysis in the laboratory. In this way, our survey strategy comprises the full range of spatial scales, from kilometre to sub-centimetre.

The total magnetic field map obtained from fixed-wing UAS data in Silinjärvi shows a north-south trending positive magnetic anomaly in the central part of the survey area, and smaller anomalies in the east (Fig. 4C). The central anomaly coincides well with structural features from the regional geological map (see "Bedrock of Finland"; https:// gtkdata.gtk.fi/Kalliopera/index.html) and is described as a major fault zone with strong deformation trends (Salo 2016). O'Brien $e$ al. (2015) described the zone as an interface of glimmerite-carbonatite - present as fine and elongated carbonatite lenses in a phlogopite matrix - and surrounding 
fenites. Although carbonatites and fenites at the open test outcrops did not show diagnostic spectral absorptions in the spectral range of the hyperspectral camera used here, separation of the HSI mosaic in the minimum noise fraction plot is indicative of the different lithologies (Fig. 4D). Field validation was achieved by finding characteristic HSI features that are associated with carbonatite bearing units (Fig. 4D) from ground-based spectroradiometry, thin-section microscopy and mineralogy and geochemistry from rock samples (unpublished data, Robert Jackisch).

Future work will investigate how information from all datasets - both field validation and in particular from the UAS surveys - can be integrated to gain an improved understanding of the relationships between magnetic and hyperspectral properties and rock composition and, hence, surface geology and mineral deposit characteristics. We plan to deploy our UAS based systems to an exploration target in the remote, Arctic environment of Greenland in the summer of 2019. Finally, we will integrate a multispectral sensor on a fixed-wing UAS to capture multispectral information over larger areas of several kilometres within a single flight.

\section{Acknowledgements}

The MULSEDRO project is funded by EIT RawMaterials (project number: 16193). We thank Aleksi Salo (Yara Suomi Oy) for allowing us to undertake surveys within YARA's premise. Comments from the reviewers Bo Møller Stensgaard and Alireza Malehmir improved the manuscript.

\section{References}

Benecke, N., Born, A., Boerner, A., Rapp, S., Stelzer, P., Tsirigotis, N., Weber, M. \& Zuev, S. 2016: Mobile solution for positioning, 3D-mapping and inspection in underground mining. $16^{\text {th }}$ International Congress for mine surveying, Brisbane, Australia. 12-16 Sept. 2016.

Colomina, I. \& Molina, P. 2014: Unmanned aerial systems for photogrammetry and remote sensing: A review. ISPRS Journal of Photogrammetry and Remote Sensing 92, 79-97. https://doi.org/10.1016/j. isprsjprs.2014.02.013

Cunnigham, M., Samon, C., Wood, A. \& Cook, I. 2018: Aeromagnetic surveying with a rotary-wing unmanned aircraft system: a case study from a zinc deposit in Nash Creek, New Brunswick, Canada. Pure Ap- plied Geophysics 175, 3145-3158. https://doi.org/10.1007/s00024017-1736-2

Dentith, M. \& Mudge, S.T. 2014: Geophysics for the mineral exploration geoscientist. Cambridge University Press. https://doi.org/10.1017/ cbo9781139024358

Gupta, R.P. 2018: Geobotanical guides. In: Remote Sensing Geology. Third edition, Springer-Verlag, Berlin, 347-348. https://doi. org/10.1007/978-3-662-55876-8_19

Jackisch, R., Lorenz, S., Zimmermann, R., Möckel, R. \& Gloaguen, R. 2018: Drone-borne hyperspectral monitoring of acid mine drainage: an example from the Sokolov Lignite District. Remote Sensing 10, 385. https://doi.org/10.3390/rs10030385

Jakob, S., Zimmermann, R. \& Gloaguen, R. 2017: The need for accurate geometric and radiometric corrections of drone-borne hyperspectral data for mineral exploration: MEPHySTo-a toolbox for pre-processing drone-borne hyperspectral data. Remote Sensing 9, 88. https://doi. org/10.3390/rs9010088

Kirsch, M., Lorenz, S., Zimmermann, R., Tusa, L., Möckel, R., Hödl, P., Booysen, R., Khodadadzadeh, M. \& Gloaguen, R. 2018: Integration of terrestrial and drone-borne hyperspectral and photogrammetric sensing methods for exploration mapping and mining monitoring. Remote Sensing 10, 1366. https://doi.org/10.3390/rs10091366

Malehmir, A., Dynesius, L., Paulusson, K., Paulusson, A., Johansson, H., Bastani, M., Wedmark, M. \& Marsden, P. 2017: The potential of rotary-wing UAV-based magnetic surveys for mineral exploration: A case study from central Sweden. The Leading Edge, 552-557. https://doi. org/10.1190/tle36070552.1

Salo, A. 2016. Geology of the Jaakonlampi area in the Siilinjärvi carbonatite complex. 30 pp. Bachelor's thesis. University of Oulu, Finland.

O’Brien, H., Heilimo, E. \& Heino, P. 2015: The Archean Siilinjärvi Carbonatite Complex. In: Maier, W., O’Brien, H. \& Lahtinen, R. (eds): Mineral deposits of Finland, 327-343. Elsevier. https://doi. org/10.1016/b978-0-12-410438-9.00013-3

Pirttijärvi M. 2003: Numerical modelling and inversion of geophysical electromagnetic measurements using a thin plate model. $\mathrm{PhD}$ thesis, University of Oulu, Finland.

\section{How to cite}

Heincke, B., Jackisch, R., Saartenoja, A., Salmirinne, H., Rapp, S., Zimmermann, R., Pirttijärvi, M., Sörensen, E.V., Gloaguen, R., Ek, L., Bergström, J., Karinen, A., Salehil, S., Madriz, Y., Middleton, M. 2019: Developing multi-sensor drones for geological mapping and mineral exploration: setup and first results from the MULEDRO project. Geological Survey of Denmark and Greenland Bulletin 43, e2019430302.https://doi.org/10.34194/GEUSB-201943-03-02

\footnotetext{
${ }^{*}$ Corresponding author: Björn Heincke |E-mail:bhm@geus.dk

${ }^{1}$ Department of Glaciology and Climate, Geological Survey of Denmark and Greenland (GEUS), Øster Voldgade 10, DK-1350, Copenhagen K, Denmark.

${ }^{2}$ Helmboltz-Zentrum Dresden-Rossendorf, Helmholtz Institute Freiberg for Resource Technology, Chemnitzer Str. 40, 09599 Freiberg, Germany

${ }^{3}$ Radai Oy, Teknologiantie 18, 90590 Oulu, Finland

${ }^{4}$ Geological Survey of Finland, Lähteentie 2, FI-96101 Rovaniemi, Finland

${ }^{5}$ Deutsche Montan Technologie GmbH, Am Technologiepark 1, 45307 Essen, Germany

${ }^{6}$ LTU Business AB, Aurorum 1, 97775 Luleå, Sweden
} 